\title{
Use of levitating liquid micro-droplets as tracers to study the evaporation in the vicinity of the contact line
}

\author{
Dmitry Zaitsev ${ }^{1,2^{*}}$, Dmitry Kirichenko ${ }^{1,3}$, and Oleg Kabov ${ }^{1,2}$ \\ ${ }^{1}$ Institute of Thermophysics, 630090 Novosibirsk, Russia \\ ${ }^{2}$ National Research Tomsk Polytechnic University, 634050 Tomsk, Russia \\ ${ }^{3}$ Novosibirsk State University, 630090 Novosibirsk, Russia
}

\begin{abstract}
Self-organization of a cloud of liquid micro-droplets into an ordered two-dimensional array, levitating over a heated layer of liquid due to upward vapor flow, has been observed in several recent experimental works. In the present paper, the levitating micro-droplet array is studied under the condition when the liquid layer ruptures and a dry spot form on the heater. It has been found, that when the levitating micro-droplets are moving from wetted heater area to dry heater area and passing over the contact line, the micro-droplets levitation height is drastically increased, which is indicative of the intensive evaporation occurring in the region of the contact line.
\end{abstract}

\section{Introduction}

A spatially ordered structure of micro-droplets levitating above the free surface of a liquid layer spot-heated from below was discovered in [1]. The array consists of several of hundreds of drops packed into one layer, forming a hexagonal structure. The drop diameter is on the order of $10 \mu \mathrm{m}$; the distance between the drops is of several drop diameters, while the drops levitation height above the liquid surface is comparable to the drop size. The possible mechanism of micro-drops levitation is the Stokes force acting onto a drop from the vapor flow arising from the heated liquid surface [2, 3]. Figure 1 shows a typical image of levitating micro-droplet array over a heated layer of liquid, obtained in the present study.

Among the effective solutions for removal of high heat fluxes, there are systems of spray cooling $[4,5]$ as well as systems using stratified two-phase flows in micro-/minichannels $[6,7]$. Intensive evaporation from the contact line region can be a key factor for the efficiency of these systems. In spray cooling, the impact and subsequent spreading of droplets on the surface results in formation of multiple contact lines. Strong correlation between the heat transfer rate and the contact line length per unit area is observed $[5,8]$. In stratified two-phase flows, the highest heat transfer rate is reached in the regime when local dry areas on the heater quickly appear and disappear [9], thus forming multiple contact lines.

*Corresponding author: zaitsev@itp.nsc.ru 
In this paper the levitating micro-droplet array is used as natural tracers to study the evaporation in the vicinity of the contact line at the boundary of a dry spot formed in a horizontal water layer heated from below. A relatively large heater of size $10 \times 10 \mathrm{~mm}^{2}$ is used (in papers [1-3] the size of the heater was around $1 \mathrm{~mm}$ ). With the help of high-speed imaging technique, we study the behaviour of the droplet array as it approaches and passes over the contact line region.

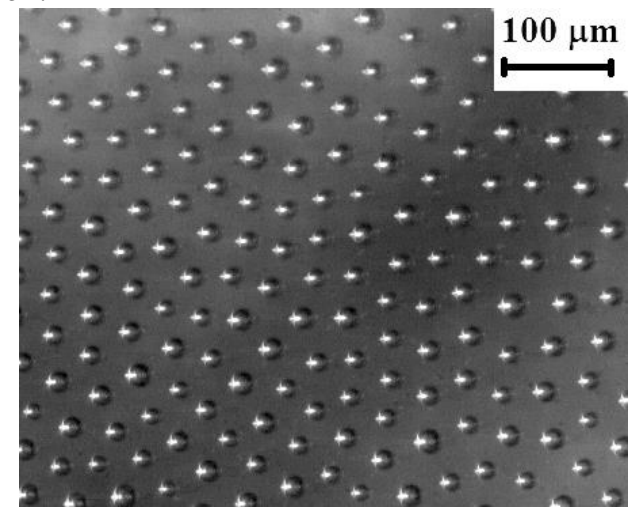

Fig. 1. Top view of an array of levitating micro-droplets, formed over a heated layer of water (heater of size $10 \times 10 \mathrm{~mm}^{2}$, heat flux of $27 \mathrm{~W} / \mathrm{cm}^{2}$, water layer thickness of $0.4 \mathrm{~mm}$ ).

\section{Experimental setup}

Schematic and photograph of the experimental setup are presented in Fig. 2. The test section is a stainless steel plate $40 \times 40 \times 5 \mathrm{~mm}^{3}$ in size with a flush-embedded $10 \times 10 \mathrm{~mm}^{2}$ copper rod, electrically heated by a nichrome wire. A layer of insulation material (mineral wool) is superimposed over the wire (nichrome wire with insulation material are not shown in Fig.2). The heater surface temperature is measured at several points by thermocouples with the accuracy of $0.1 \mathrm{~K}$ and frequency of $1 \mathrm{~Hz}$. According to the measurements, the condition $\mathrm{T}_{\mathrm{w}}=$ const is always met along the copper surface. In the experiments, $\mathrm{T}_{\mathrm{w}}$ was varied from 75 to $100{ }^{\circ} \mathrm{C}$. The heat flux is determined by the electric power released at the nichrome wire. Ultra-pure degassed distilled water (Merck Millipore) with the initial temperature of $25^{\circ} \mathrm{C}$ is used as the working liquid. The test section is installed horizontally and open to the atmosphere; the temperature of ambient air is $25 \pm 2{ }^{\circ} \mathrm{C}$.

The working surface of the test section was mechanically polished. The morphology of the surface was analysed using scanning electron microscope (JEOL JSM6700F) and atomic force microscope (Solver Pro NT MDT), Fig.3. The root mean square (RMS) surface roughness, measured on a $22 \mu \mathrm{m}^{2}$ area of the heater surface, was found to be 500 $\mathrm{nm}$, with the average roughness of $0.394 \mathrm{~nm}$. Thus, the roughness of the working surface is negligible compared to typical micro-droplet size $(10-30 \mu \mathrm{m})$ and typical micro-droplet levitation height above the dry substrate (5-15 $\mu \mathrm{m}$, see below).

The equilibrium contact angle on the copper surface was measured at different points by the method of a sessile liquid drop at the room temperature of $25 \pm 2{ }^{\circ} \mathrm{C}$. To obtain the profile of the drop surface, the shadow method was used with a collimated light source and digital camera Nikon D800 having resolution of 6 micron/pixel. The resulting images were processed using the software by KRUSS. The advanced static contact angle measured at different points over the surface of the copper heater was $74 \pm 9^{\circ}$, whereas the receding contact angle was close to zero $\left(<10^{\circ}\right)$. 


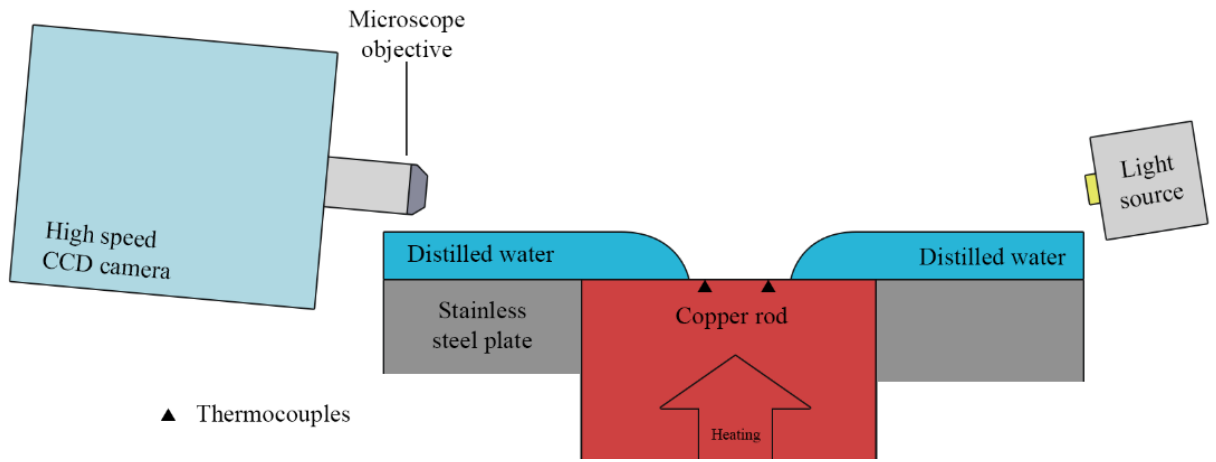

(a)

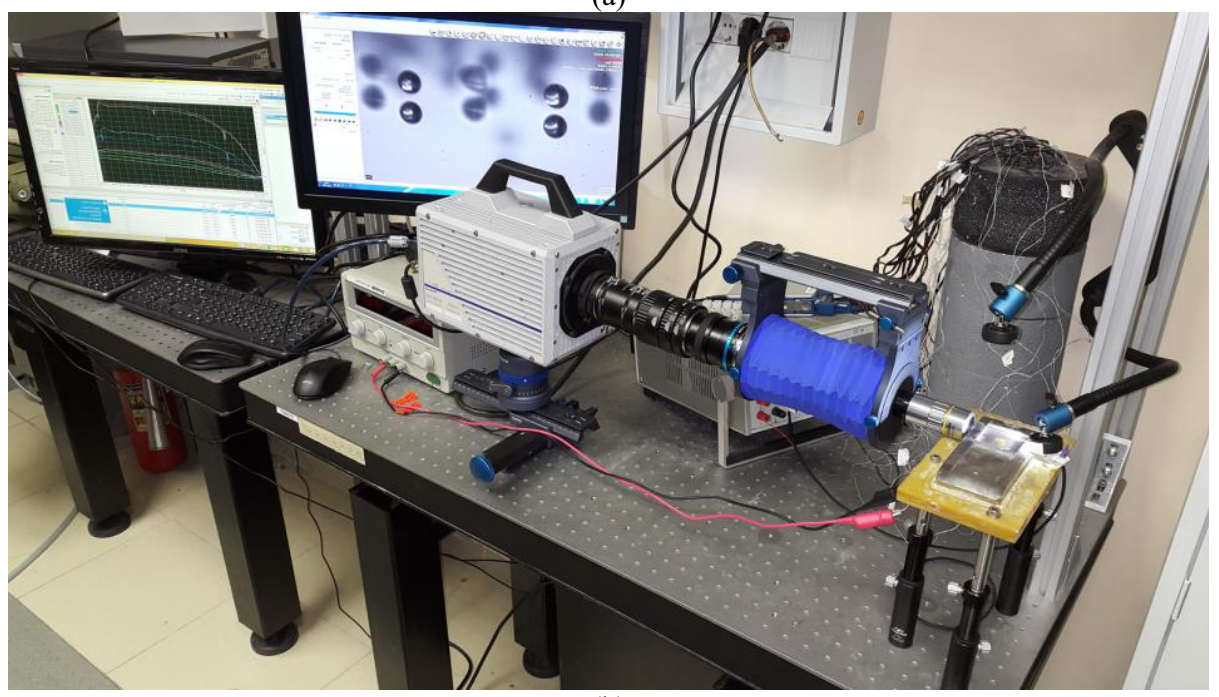

(b)

Fig. 2. Schematic (a) and photograph (b) of the experimental setup.

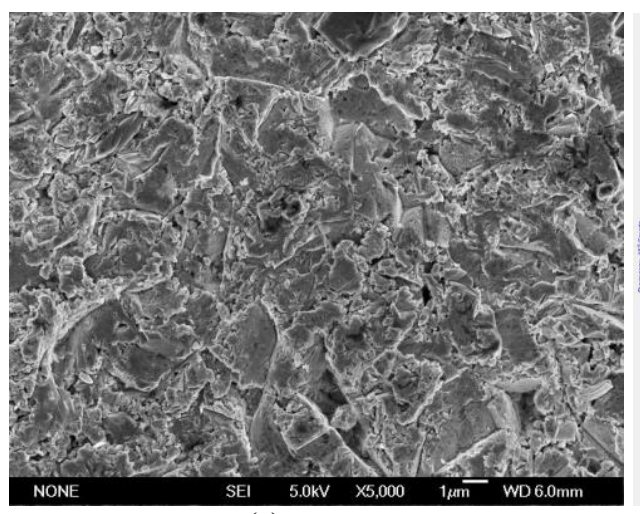

(a)

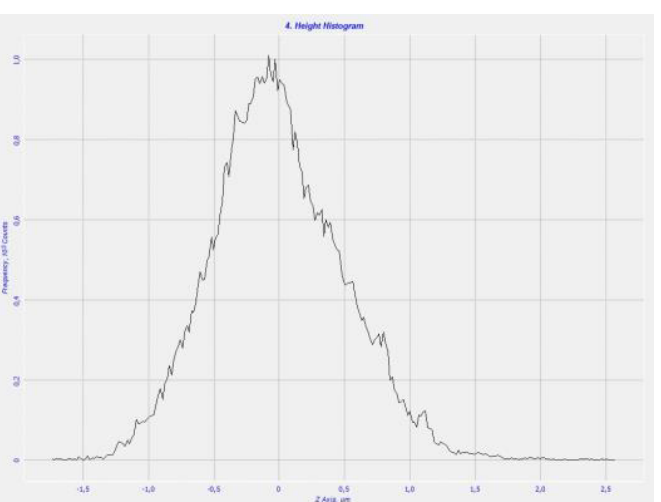

(b)

Fig. 3. Image of the heater surface obtained using scanning electron microscope (a), and height histogram obtained using atomic force microscope (b).

Optical recording was made with a high-speed camera FASTCAM SA1.1 (5600 fps with resolution of $1024 \times 1024$ pixel) coupled with a microscope objective through a set of 
extension tubes and bellows. Field of view of the camera was $800 \times 800 \mu \mathrm{m}^{2}$ (resolution of $0.78 \mu \mathrm{m}$ per pixel). The optical axis of the camera was aligned at an angle of $5^{\circ}$ with respect to the horizon. The experimental procedure is as follows:

1) A predetermined volume of the working liquid is deposited with a syringe onto the working surface to form a liquid layer with the initial thickness of $400 \mu \mathrm{m}$.

2) With the help of an air jet a dry spot (about $1 \mathrm{~mm}$ in size) is formed on the copper surface.

3) The optical system is focused onto the contact line at the edge of the dry spot.

4) The heater is switched on, which induces formation of micro-drops levitating over the heated layer of liquid.

5) When the levitating micro-drops move to the dry spot, the recording is started.

\section{Experimental results}

Increase of the heat flux induces formation of the array of micro-drops levitating over the heated liquid layer (typical top view is shown in Fig. 1). Usually, the array was of an elliptical shape with the size varying approximately from 500 to $4000 \mu \mathrm{m}$. In most of previous studies [1-3] the micro-droplet array was localized due to the use of localized heating (the size of the heater was around $1 \mathrm{~mm}$ ), and as such the size of the array roughly coincided with the size of the heater. In contrast, we use a heater having size much larger than typical size of the cluster, which allows us to obtain larger arrays of droplets.

Upon the formation of a dry spot in the liquid layer, the micro-droplets move down the slope of the interface under the action of gravity toward the dry area. As they get close to the contact line, the height of their levitation increases several times and eventually the micro-droplets turn out to be levitating over the heater surface, Fig. 4. After that, they disappear by evaporation within a second or so while continuing to levitate over the dry substrate.

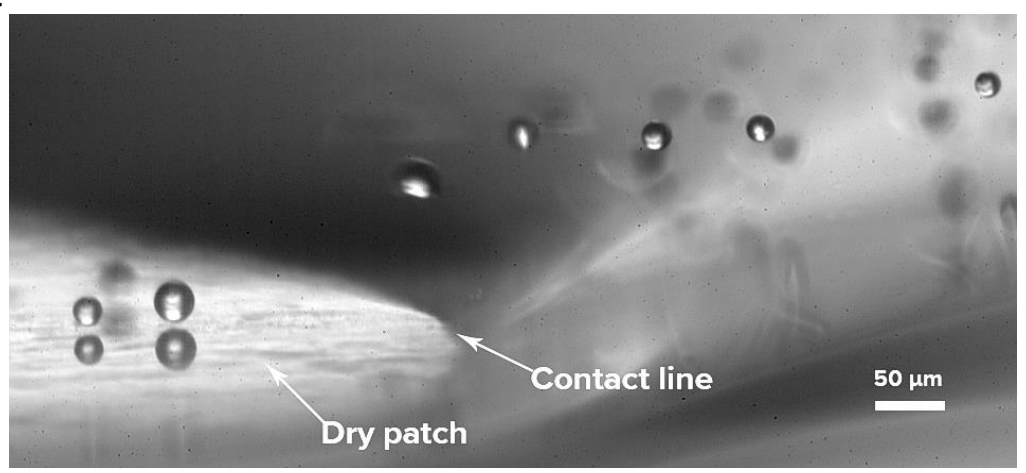

Fig. 4. Side view of micro-droplets flying over the contact line (substrate temperature of $95^{\circ} \mathrm{C}$ ).

We revealed three common scenarios of micro-droplets transition from the wetted area to the dry area. First, a droplet can simply fly over the contact line with substantial increase of its levitation height, as depicted in Fig. 5a. The result shown in Fig. 5a was obtained for a droplet of diameter of $16 \mu \mathrm{m}$ and substrate temperature of $79{ }^{\circ} \mathrm{C}$. The second common scenario is when the droplet is following a circular-like trajectory in the vicinity of the contact line, with one or more full rotations, before eventually getting out of this cycle and flying to the dry area, Fig. $5 \mathrm{~b}$ (droplet size is $20 \mu \mathrm{m}$, substrate temperature is $81{ }^{\circ} \mathrm{C}$ ). This scenario can be connected with the vortex flow of the gas-vapor mixture formed near the contact line. Finally, some droplets can be levitating at a fixed location above the contact line, as shown in Fig. $5 \mathrm{c}$ (droplet diameter is $26 \mu \mathrm{m}$, substrate temperature is $97^{\circ} \mathrm{C}$ ). In this 
case, the dynamic force of the air-vapor flow from the contact line is balanced with the gravity force acting on the droplet. We would like to emphasize that while actual snapshots, shown in Fig. 5, were made at slightly different conditions, all three scenarios were observed under a wide variety of substrate temperatures (from 75 to $100{ }^{\circ} \mathrm{C}$ ).

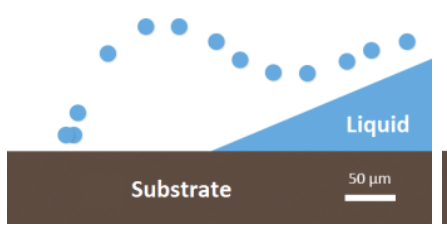

(a)

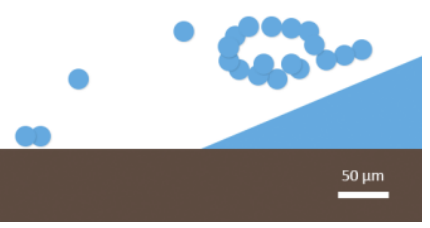

(b)

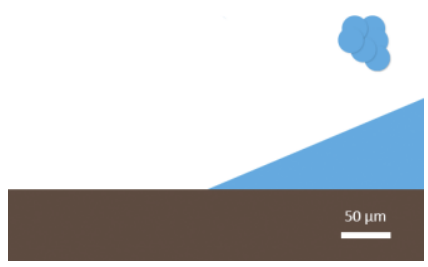

(c)

Fig. 5. Three common scenarios of micro-droplets transition from wetted area to dry area: simple flyover above the contact line (a), fly-over with circular trajectory (b), and levitating at a fixed location (c). Positions of micro-droplets are shown at equal time intervals of $0.02 \mathrm{~s}$. Micro-droplets are drawn in the scale.

\section{Conclusions}

Levitating micro-droplets, formed in the upward vapor flow above the heated liquid surface, are used as tracers to study the evaporation in the vicinity of the contact line. It has been found, that while the levitating micro-droplets are passing from the wetted substrate area over the contact line to the dry substrate area, the droplets levitation height is increased several times. From this we conclude that the specific evaporation rate in the vicinity of the contact line must be several times higher than that at a distance from the contact line. Three common micro-droplet trajectories are identified: 1) "simple" fly-over, 2) fly-over with circular motion, and 3) nearly steady-state levitation.

This work was supported by the Russian Science Foundation, project No. 1419-01755.

\section{References}

1. A.A. Fedorets, JETP Lett. 79, 372 (2004)

2. A.A. Fedorets, I.V. Marchuk and O.A. Kabov, Tech. Phys. Lett. 37, 116 (2011)

3. A.A. Fedorets, I.V. Marchuk and O.A. Kabov, JETP Lett. 99, 266 (2014)

4. B. Horacek, K.T.Kiger, J. Kim, Int. J. Heat Mass Tran. 48, 1425 (2005)

5. J. Kim, Int. Journal Heat Fluid Flow 28, 753 (2006)

6. O.A. Kabov, Yu.V. Lyulin, I.V. Marchuk, D.V. Zaitsev, Int. J. Heat Fluid Fl. 28, 103 (2007)

7. Zaitsev D.V., Rodionov D.A. and Kabov O.A. Tech. Phys. Lett. 35, 680 (2009)

8. A.A. Semenov, D.V. Feoktistov, D.V. Zaitsev, G.V. Kuznetsov, O.A. Kabov Thermophys. Aeromech. 22, 771 (2015)

9. O.A. Kabov, D.V. Zaitsev, V.V. Cheverda, A. Bar-Cohen, Exp. Therm Fluid Sci. 35, 825 (2011) 